Pirineos, 164: 69 a 92, JACA; 2009. ISSN 0373-2568

\title{
MEDIO SIGLO DE CAMBIOS PAISAJÍSTICOS EN LA MONTAÑA MEDITERRÁNEA. PERCEPCIÓN Y VALORACIÓN SOCIAL DEL PAISAJE EN LA ALTA GARROTXA (GIRONA)
}

\author{
J. Vila Subirós, A. Ribas Palom, D. Varga linde \& A. Llausàs Pascual \\ Unitat de Geografía. Institut de Medi Ambient. Universitat de Girona. Cataluña (España). \\ josep.vila@udg.es
}

\begin{abstract}
Rural depopulation and abandonment of farming activities have resulted in an intense transformation of the characteristic landscapes of Mediterranean mountains. A dynamic characterized by an intense process of expansion of forested cover in detriment to livestock and agricultural areas. This process, which produces effects such as biodiversity and cultural heritage loss and contributes to the spread of wildfires, can be mapped, quantified and described with high accuracy through the means of digital mapping, geographic information systems and landscape indexes. But what is the perception and valuation of these changes by the stakeholders involved in the management of these territories? This article attempts to answer this question in the protected area of Alta Garrotxa (Girona), where a strong correlation between landscape dynamics and their perception by the stakeholders is revealed. On the other hand, the valuation and future prospects produce diverse and often contradictory points of views that illustrate the existing difficulties to management.
\end{abstract}

Keywords: mediterranean mountain, landscape change, landscape ecology, stakeholders' landscape perception, Alta Garrotxa.

RESUMEN.- El despoblamiento rural y el abandono de la actividad agraria han originado una intensa transformación de los paisajes característicos de las montañas mediterráneas. Una dinámica marcada por un intenso proceso de incremento de la superficie forestal en detrimento de las zonas dedicadas a la ganadería y a la agricultura. Este proceso, que repercute en aspectos tan variados como la pérdida de biodiversidad y patrimonio cultural o la proliferación de incendios forestales, puede ser cartografiado, cuantificado y caracterizado con una gran exactitud gracias a la cartografía digital, los sistemas de información geográfica y los índices de paisaje. ¿Pero cuál es la percepción y la valoración de estos cambios por parte de los agentes sociales 
J. VILA SUBIRÓS, A. RIBAS PALOM, D. VARGA LINDE \& A. LLAUSÀS PASCUAL

involucrados en la gestión de estos territorios? En este artículo se intenta dar respuesta a esta pregunta en el espacio protegido del Alta Garrotxa (Girona), donde se pone de manifiesto una elevada coincidencia entre las dinámicas del paisaje y la percepción de estas dinámicas por parte de los agentes sociales. En cambio la valoración social de estos cambios y las perspectivas de futuro generan opiniones diversas y a menudo contradictorias que ilustran las dificultades de gestión existentes.

Palabras clave: montaña mediterránea, cambios paisajísticos, ecología del paisaje, percepción social del paisaje, Alta Garrotxa.

\section{Introducción}

Los cambios sociales, económicos, demográficos y culturales que conoce la montaña mediterránea desde medianos del siglo XX y hasta nuestros días, vinculados principalmente al despoblamiento rural y el abandono de la actividad agraria, han generado un galopante proceso de homogeneización del paisaje. El concepto de homogeneización del paisaje se interpreta como el proceso dinámico caracterizado por el aumento de un determinado tipo de hábitats en detrimento de otros. Este proceso genera una progresiva uniformidad del complejo mosaico paisajístico que, como consecuencia, comporta la pérdida de la heterogeneidad de hábitats históricamente característica de la montaña mediterránea bajo el influjo de formas tradicionales de gestión (BLONDEL \& ARONSON, 1999; GROVE \& RACKMAN, 2001).

La evolución, los procesos, los potenciales escenarios de futuro y especialmente los efectos derivados de este proceso de homogeneización paisajística en la montaña mediterránea son múltiples y han sido descritos y analizados ampliamente en diversos casos de estudio (FARINA et al., 2005; LASANTA et al. 2005 y 2006; VARGA \& VILA, 2006). Por una parte se han constatado los efectos negativos de este proceso sobre la diversidad biológica fruto de la pérdida, degradación, fragmentación y homogeneización de hábitats a consecuencia de los cambios experimentados por la progresiva desaparición de la actividad humana en estos espacios (SWIHART \& MOURE, 2004). En el caso concreto de los hábitats propios de la montaña mediterránea, la homogeneización paisajística provoca, por ejemplo, la disminución de la cantidad absoluta y relativa de zonas de ecotono y, por el contrario, favorece el aumento del denominado hábitat interior. Este cambio morfológico provoca que diferentes grupos de organismos respondan de manera distinta, favoreciendo a las especies de hábitat interior en detrimento de las especies de margen o ecotono (FARINA, 1997). También se ha comprobado que aumenta la probabilidad de extinción de especies a causa de la estocasticidad demográfica, entendida 
como las fluctuaciones naturales en el número de individuos, distribución en clases de edad y razón sexual —número de hembras y machos— de la población, que comporta variaciones en sus tasas de supervivencia y reproducción. En la misma línea influye el deterioro genético de las especies con poca capacidad para desplazarse por hábitats no forestales, ya que el intercambio de individuos entre las distintas zonas de pasto y cultivos aislados por masa forestal es menor (FORMAN \& GODRON, 1986; FORMAN, 1995).

Por otra parte, el abandono de la actividad humana en estos espacios de montaña mediterránea contribuye al desarrollo de los estratos verticales de la vegetación, así como a su crecimiento horizontal, a la aparición de especies leñosas y a la acumulación de materia vegetal en descomposición. En consecuencia, estos espacios abandonados resultan más vulnerables al inicio y propagación de los incendios forestales (BOADA, 2003). La heterogeneidad del paisaje constituye sin duda alguna un instrumento crucial para reducir la velocidad de propagación de los incendios forestales y facilitar la accesibilidad para extinguirlos. Asimismo, el progresivo dominio de los hábitats forestales en detrimento de los no forestales repercute en la modificación de los regímenes hidrológicos y, en consecuencia, en la disponibilidad de los recursos hídricos (BEGUERÍA et al., 2003; DEFRIES \& ESHLEMAN, 2004; CONSANDEY et al., 2005 y OTERO et al., 2008). Otros efectos negativos relacionados con los procesos de despoblación y homogeneización del paisaje de la montaña mediterránea serían la degradación de su rico patrimonio cultural (casas rurales, yacimientos arqueológicos, iglesias, caminos, etc.), la pérdida del conocimiento popular asociado a las actividades económicas tradicionales de cada área en cuestión (carboneo, caza, recolección de plantas medicinales, frutos u otras prácticas humanas) y, en general, la disminución de la calidad paisajística de los espacios que sufren estos procesos de abandono (LASANTA, 1988; LASANTA et al., 2005 y 2006; VARGA \& VILA, 2006).

Este artículo tiene por objetivo poner en evidencia la controvertida relación entre los cambios ocurridos en el paisaje de la montaña mediterránea y la percepción y valoración que de estos cambios y sus efectos realizan los distintos agentes sociales más directamente involucrados en su gestión. Las diferentes percepciones sobre estos cambios y de forma especial la distinta valoración de los agentes sociales en relación a las transformaciones que ha vivido el paisaje, suponen al mismo tiempo una interpretación diferencial sobre el modelo de gestión y las prioridades de intervención. Estas visiones desiguales sobre las necesidades y las fórmulas de actuación es una de las bases que explica las dificultades y las tensiones en relación a la definición de un modelo de gestión consensuado. Un adecuado conocimiento sobre estas dificultades inherentes a la percepción y valoración de los cambios paisajísticos es un primer paso para afrontar un modelo de gestión más integrador de las 
distintas sensibilidades. Estos objetivos se plantean a partir del análisis del caso concreto de la Alta Garrotxa, un espacio natural protegido de la montaña media mediterránea situado en los Prepirineos orientales. Una nueva aportación, en este caso desde el contexto del mediterráneo, a las variadas investigaciones que desde diferentes ópticas y perspectivas buscan la incorporación de la dimensión perceptiva en el análisis y valoración de la evolución de los paisajes (LEWIS, 2007; HUNZINKER et al., 2008)

El artículo se estructura en cuatro partes claramente diferenciadas. En un primer momento se presenta el área de estudio que constituye la Alta Garrotxa a partir de una síntesis de sus principales características físicas y socioeconómicas. A continuación se detallan las distintas metodologías utilizadas para conocer los cambios experimentados en su paisaje en el período 1957-2007 y a la vez evaluar e interpretar la percepción que los distintos agentes sociales realizan de estos cambios. Por último se presentan los resultados obtenidos de la aplicación de estas dos metodologías y se extraen las correspondientes conclusiones.

\section{Presentación del área de estudio: la Alta Garrotxa}

La Alta Garrotxa ocupa 32686 ha de extensión y está protegida como espacio de interés natural por el Pla d'Espais d'Interès Natural de Catalunya (PEIN, Decreto 328/1992 de 14 de diciembre) equivalente a la categoría VI de la UICN. También recientemente (Orden del Departament de Medi Ambient i Habitatge (DMAH) de 1 de marzo de 2005) ha sido declarada Zona de Especial Protección para las Aves (ZEPA) y por tanto un espacio clave dentro de la red Natura 2000 y Lugar de Importancia Comunitaria (LIC). La abundancia de pliegues y fallas ha configurado un relieve muy complejo que ha dado nombre a este territorio (las "garrotxes" son, según la elocuente expresión catalana, "terres aspres i de mala petja"), de tipo calcáreo, con un gradiente altitudinal que va de los prácticamente 200 metros a los $1558 \mathrm{~m}$ de la cima más eleva$\mathrm{da}$, el Comanegre. Las temperaturas son relativamente suaves (la temperatura media anual oscila entre los $8^{\circ} \mathrm{C}$ de las zonas septentrionales elevadas más frías y los $14^{\circ} \mathrm{C}$ del área meridional más templada) y la precipitación considerable aunque con una importante amplitud diferencial (varía entre 800 del extremo sudoriental y $1200 \mathrm{~mm}$ anuales de la parte noroccidental).

La comunidad vegetal dominante es el encinar y, a medida que se gana altitud, aparecen los robledales de roble pubescente y en las vertientes umbrías el hayedo. En conjunto, la superficie forestal ocupa aproximadamente el 90\% del territorio. Faunísticamente dominan las especies vinculadas a los espacios forestales (Genetta genetta, Felis sylvestris...) con una especial rele- 


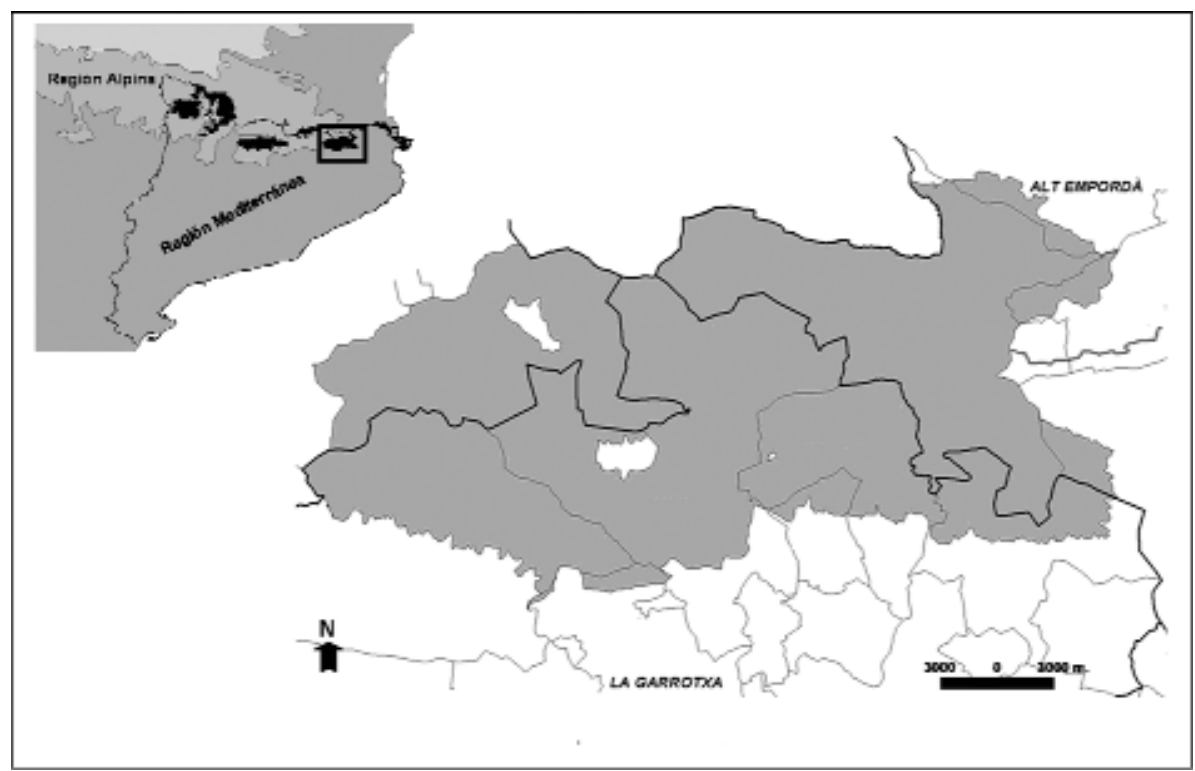

Figura 1: Localización de la Alta Garrotxa. Figure 1. Location of the "Alta Garrotxa".

vancia de los ungulados salvajes como Capreolus capreolus, Ovis musimon o Sus scrofa. (PAGÈS \& ANGLADA, 2008). Una atención especial merece la ornitofauna donde destacan las especies que han determinado su reciente declaración como Zona de Especial Protección para las Aves (Aquila chrysaetos, Pernis apivorus, Alcedo atthis, Bubo bubo, Lanius collurio, Dryocopus martius). El progresivo aumento de la superficie forestal ha comportado que especies como el águila real (Aquila chrysaetos) padezca graves dificultades de reproducción en buena parte fruto de esta dinámica del paisaje (FABREGÓ et al. 2008), mientras que otras especies como el búho real (Bubo bubo) se han situado al borde de su extinción local (TRABALÓN, 2008).

La persistencia de un rico patrimonio cultural (yacimientos arqueológicos, casas rurales, iglesias y ermitas, etc.), repartido por toda la Alta Garrotxa, atestigua la importancia que tuvieron antaño los asentamientos humanos y las actividades agrícolas y ganaderas en esta zona. El despoblamiento y abandono de este territorio ha sido especialmente intenso desde mediados del pasado siglo, a causa básicamente de la desvalorización del carbón vegetal como recurso energético y por el atractivo que supuso para sus habitantes la actividad industrial localizada en el valle del río Fluvià. De los 1302 habitan- 
tes censados en 1950 se pasa a los 304 del 2001 (con un mínimo histórico de 185 habitantes en el año 1986), lo que supone una reducción cercana al 75\% en poco más de 50 años. Las pocas actividades económicas existentes actualmente giran alrededor del turismo rural, el senderismo y algunos reductos de actividad forestal, ganadera y agrícola por parte de la población local.

\section{Metodología y fuentes documentales utilizadas}

En este apartado se presenta la metodología y las fuentes documentales utilizadas en:

1) La caracterización de los principales cambios paisajísticos ocurridos en la Alta Garrotxa en los últimos 50 años a partir del análisis de los cambios en los usos y cubiertas del suelo.

2) El análisis de la percepción y valoración que de estos cambios y de sus efectos realizan los distintos agentes sociales del territorio.

En el primer caso se toma como área de estudio de referencia el espacio constituido por los valles de Hortmoier y Sant Aniol, con una superficie total 5140 ha. Esta zona aglutina sin duda alguna las mejores muestras de las características territoriales y paisajísticas del Alta Garrotxa. En el segundo caso, y tal y como se expondrá más adelante, el área de estudio de referencia es el conjunto de la Alta Garrotxa, con sus 32.000 ha de superficie.

\subsection{Caracterización de las dinámicas paisajísticas (1957-2003)}

La reconstrucción y análisis del cambio en el paisaje de la Alta Garrotxa parte de la elaboración de una cartografía de los usos del suelo de los años 1957 (escala 1:33000) y 1997 (escala 1:25000) que cubre la totalidad de la Alta Garrotxa y también de una cartografía de detalle para el área piloto de estudio que es la Vall d'Hortmoier y Sant Aniol para los años 1957 (escala 1:7500) y 2003 (escala 1:5000). En el estudio de detalle la información cartográfica de 2003 se ha obtenido a partir de los ortofotografías del Instituto Cartográfico de Catalunya (ICC) a escala 1:5.000 y la realización de trabajo de campo. En cambio, la información cartográfica del año 1957 se ha obtenido de la ampliación de las fotografías aéreas del vuelo americano por parte del Servicio Geográfico del Ejército del año 1957, lo que ha permitido conseguir una cartografía a escala aproximada de 1:7500. 
Este material en formato papel se ha escaneado y ortorectificado con el software Erdas Imagine ${ }^{\circledR} 8.4$ y su módulo OrthoBase. Para la ortorectificación de las fotografías aéreas se ha utilizado un Modelo Digital de Elevaciones (MDE) ráster creado a partir del programa IsoMDE del SIG MiraMon ${ }^{\circledR}$ 5.0. La digitalización de la información de usos del suelo se ha realizado a partir de una leyenda que distingue entre las siguientes categorías de usos del suelo para cada uno de los dos años de estudio:

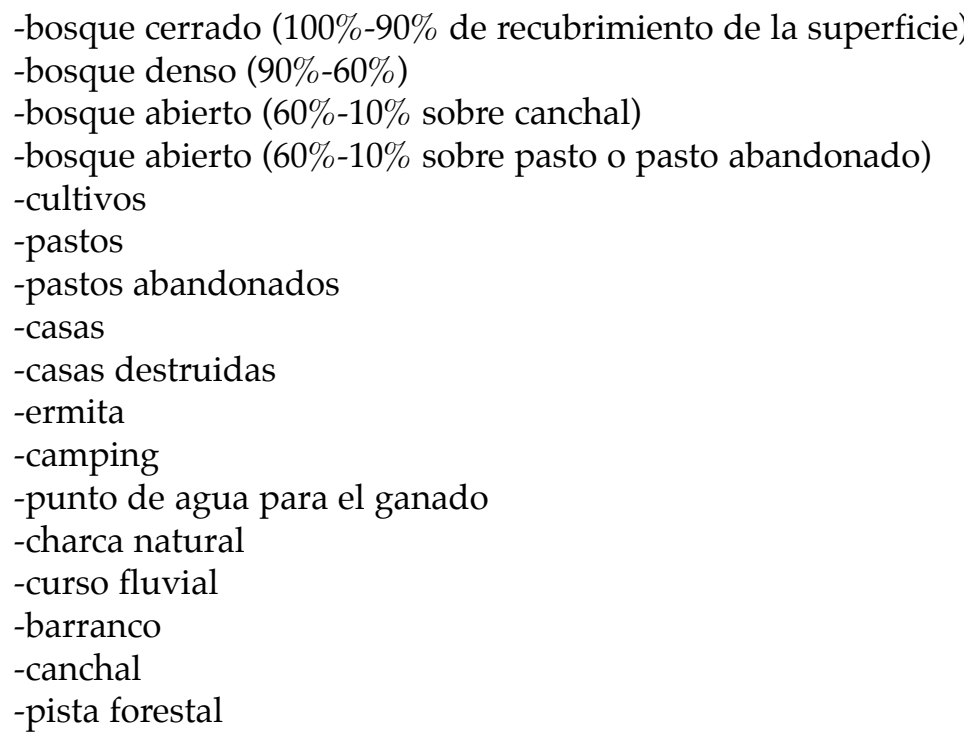

A partir de estas bases cartográficas se ha procedido a obtener los estadísticos correspondientes para cada una de ellas y de forma comparativa entre ellas. A continuación, a partir de la cartografía de usos y cubiertas del suelo elaborada anteriormente, se ha procedido a calcular diversos índices de paisaje. Los software utilizados han sido el módulo para ESRI ArcGis ${ }^{\grave{\partial}}$ Vlate $^{\mathrm{O}}$ 1.0, Patch Analyst y Fragstats. La visión e interpretación del paisaje desarrollada desde la ecología del paisaje se fundamenta en una aproximación de carácter estructural - morfológica y a la vez funcional. Los índices de paisaje aportan interesantes datos numéricos sobre la composición y la configuración de los paisajes, la proporción de cada cubierta del suelo o la superficie y forma de los elementos del paisaje. Además, los índices de paisaje permiten una útil e interesante comparación entre distintas configuraciones paisajísticas, la misma área en distintos momentos temporales o la definición de escenarios futuros (LI \& WU, 2004; MCGARIGAL \& CUSHMAN, 2005; BOTEQUILHA et al., 2006; VILA et al., 2006). 


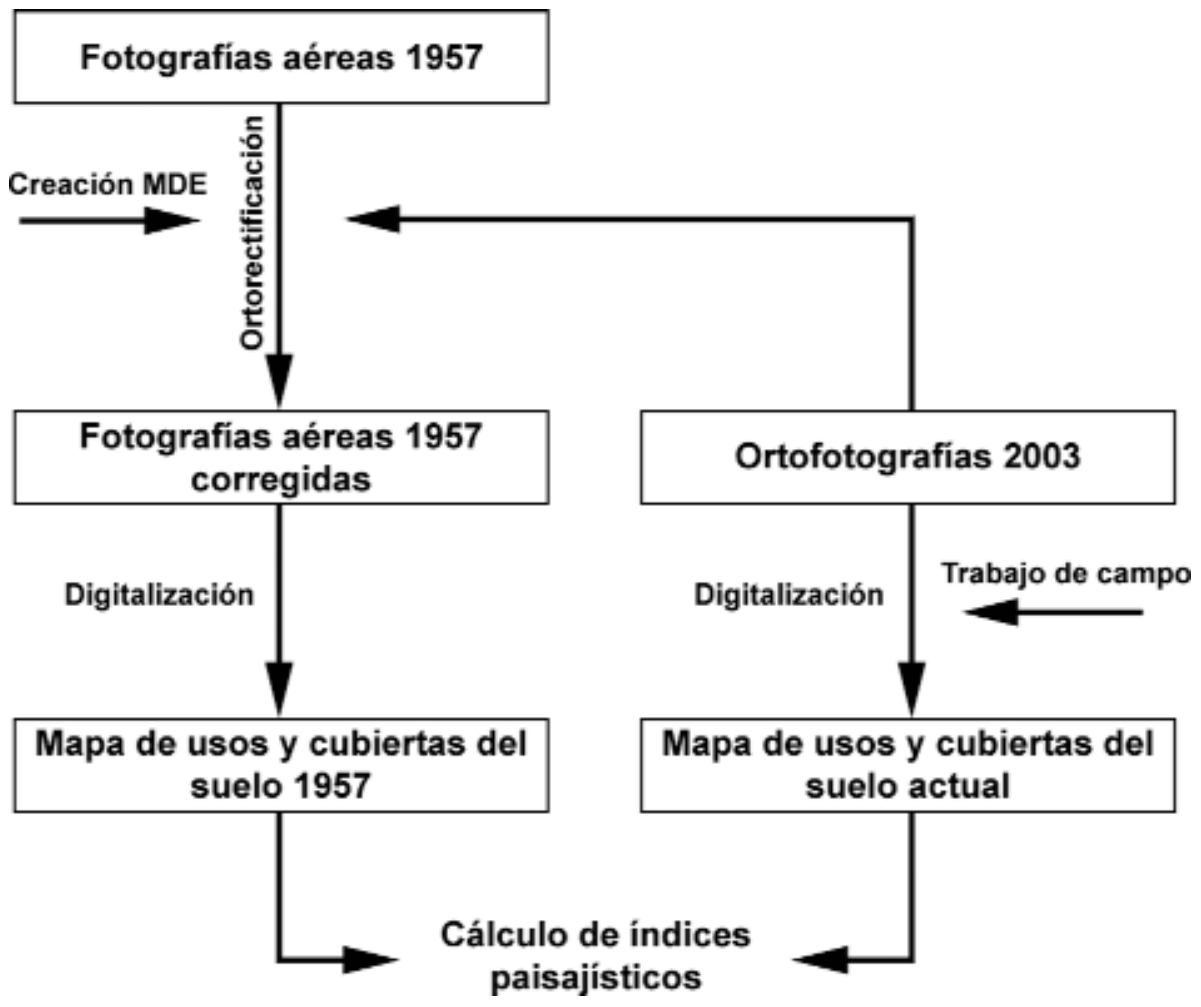

Figura 2: Esquema metodológico de generación de la cartografía y cálculo de índices paisajísticos.

Figure 2. Methodology chart to generate maps and lanscape indices calculation.

Los métodos cuantitativos en ecología del paisaje se aplican a tres niveles: fragmento, clase y paisaje. Además se pueden diferenciar cinco grandes tipos de índices de paisaje: Índices de área superficie, densidad y variabilidad; Índices de forma; Índices de ecotono y hábitat interior; Índices de distancia, vecindad y conectividad y finalmente Índices de diversidad del paisaje. No es posible analizar aquí sus características, potencialidades y limitaciones, pero existen distintas obras de referencia que las repasan detalladamente (MCGARIGAL \& MARKS, 1995; BOTEQUILHA et al., 2006; FARINA, 2006; VILA et al., 2006). 
MEDIO SIGLO DE CAMBIOS PAISAJÍSTICOS EN LA MONTAÑA MEDITERRÁNEA. PERCEPCIÓN...

3.2. Análisis de la percepción y valoración social de los cambios en el paisaje y sus efectos

Para el análisis de la percepción social de los cambios experimentados en el paisaje y sus efectos se diseñó un modelo de entrevista en profundidad (de 2 a 3 horas de duración) organizada a partir de un cuestionario que servía de guía de conversación. Este cuestionario se organizó en una batería de preguntas agrupadas entorno a seis grandes bloques temáticos:

1) Presentación del/de la entrevistado/a.

2) Características básicas de la Alta Garrotxa.

3) Descripción de las dinámicas específicas observadas en el paisaje.

4) Valoración de las dinámicas específicas observadas en el paisaje.

5) Descripción y valoración de las acciones y políticas desarrolladas

6) Visión de futuro de la Alta Garrotxa.

Se seleccionaron un total de 14 personas a entrevistar, atendiendo a la máxima representatividad posible de agentes sociales directamente involucrados en el territorio objeto de estudio. Se trata de una muestra cualitativa definida con la voluntad de recoger la opinión de representantes cualificados de cada uno de los principales sectores socioculturales implicados en el conocimiento y la gestión de este espacio natural protegido. La selección de las personas a entrevistar se determinó a partir de la experiencia acumulada por el equipo investigador después de más de una década de investigación en esta zona. Cada una de las entrevistas realizadas se registró en formato audio y fue transcrita posteriormente con el objetivo de facilitar su análisis e interpretación. En la tabla 1 se recopila el perfil de los distintos agentes sociales entrevistados a lo largo del estudio.

Tabla.1. Perfil de los agentes sociales entrevistados.

Table 1. Characterisics of the interviewee.

\begin{tabular}{|c|c|c|c|c|c|c|}
\hline & \multirow[t]{2}{*}{ TOTAL } & \multicolumn{2}{|c|}{ GÉNERO } & \multicolumn{3}{|c|}{$E D A D$} \\
\hline & & Hombres & Mujeres & $\begin{array}{c}\text { De } 18 \text { a } 40 \\
\text { años }\end{array}$ & $\begin{array}{c}\text { Entre } 40 y \\
65 \text { años }\end{array}$ & $\begin{array}{c}\text { Más de } 65 \\
\text { años }\end{array}$ \\
\hline $\begin{array}{l}\text { Agricultores y } \\
\text { ganaderos } \\
\text { autóctonos }\end{array}$ & 1 & 1 & & & 1 & \\
\hline $\begin{array}{l}\text { Agricultores y } \\
\text { ganaderos no } \\
\text { autóctonos }\end{array}$ & 2 & 2 & & 1 & 1 & \\
\hline
\end{tabular}


J. VILA SUBIRÓS, A. RIBAS PALOM, D. VARGA LINDE \& A. LLAUSÀS PASCUAL

\begin{tabular}{|c|c|c|c|c|c|c|}
\hline & \multirow[t]{2}{*}{ TOTAL } & \multicolumn{2}{|c|}{ GÉNERO } & \multicolumn{3}{|c|}{$E D A D$} \\
\hline & & Hombres & Mujeres & $\begin{array}{c}\text { De } 18 \text { a } 40 \\
\text { años }\end{array}$ & $\begin{array}{c}\text { Entre } 40 \text { y } \\
65 \text { años }\end{array}$ & $\begin{array}{c}\text { Más de } 65 \\
\text { años }\end{array}$ \\
\hline $\begin{array}{l}\text { Administración } \\
\text { pública } \\
\text { político }\end{array}$ & 1 & 1 & & & 1 & \\
\hline $\begin{array}{l}\text { Administración } \\
\text { pública } \\
\text { técnica }\end{array}$ & 1 & & 1 & 1 & & \\
\hline Propietarios forestales & 1 & 1 & & & 1 & \\
\hline Cazadores & 1 & 1 & & 1 & & \\
\hline Grupos ecologistas & 1 & 1 & & & 1 & \\
\hline Investigadores & 1 & 1 & & 1 & & \\
\hline Excursionistas & 1 & 1 & & & 1 & \\
\hline Asociaciones culturales & 1 & 1 & & & & 1 \\
\hline Artistas & 1 & 1 & & & & 1 \\
\hline Empresarios turísticos & 2 & 1 & 1 & & 2 & \\
\hline
\end{tabular}

\section{Resultados}

\subsection{Evolución de la composición y estructura paisajística en el período 1957-2003}

En este apartado se presenta un exhaustivo y detallado análisis cuantitativo de la evolución del paisaje en los valles d'Hortmoier y Sant Aniol (Alta Garrotxa). Esta zona, que se extiende por 5.140 hectáreas ubicadas en la parte central del área de estudio, es reconocida como la zona de mayor interés biogeográfico y la mejor representación de los paisajes que han singularizado este espacio natural protegido (VILA, 2000; VARGA, 2007).

La distribución de usos y cubiertas del suelo en el año 1957 se caracterizaba por una cierta proporcionalidad entre los distintos hábitats agroforestales fruto de una fuerte presión antrópica sobre los bosques. Unas masas forestales sometidas a una intensa explotación con el objetivo primordial de obtener carbón vegetal así como su transformación en pastos. En ese momento, entorno a un $45 \%$ del territorio se destina a aprovechamiento forestal y otro $45 \%$ tiene por objetivo la producción agraria, especialmente la ganadera (ver tabla 2). 
MEDIO SIGLO DE CAMBIOS PAISAJÍSTICOS EN LA MONTAÑA MEDITERRÁNEA. PERCEPCIÓN...

Tabla 2. Usos y cubiertas del suelo en el año 1957. Table 2. Land use and land cover in 1957.

\begin{tabular}{|lcc|}
\hline Usos y cubiertas del suelo & Hectáreas & $\%$ \\
\hline Bosque cerrado & 1700,41 & 33,05 \\
Bosque denso & 666,98 & 12,96 \\
Bosque abierto sobre pasto & 787,93 & 15,31 \\
Bosque abierto sobre canchal & 153,89 & 2,99 \\
Cultivos & 154,64 & 3,01 \\
Superficie denudada & 119,47 & 2,32 \\
Edificaciones & 2,45 & 0,05 \\
Pastos & 1446,51 & 28,11 \\
Pistas forestales & 85,77 & 1,67 \\
Superficie fluvial & 27,27 & 0,53 \\
\hline TOTAL & $\mathbf{5 1 4 5 , 3 4}$ & $\mathbf{1 0 0 , 0 0}$ \\
\hline
\end{tabular}

Fuente: Elaboración propia a partir de la fotografía aérea ampliada, E/ 1:7.500 del año 1957.

En el año 2003 se pone claramente de manifiesto el proceso de homogeneización paisajística que experimenta la Alta Garrotxa como consecuencia de la progresión del bosque cerrado, que llega a ocupar el $80 \%$ del área de estudio. Un dato que contrasta con el $12 \%$ al que queda limitado el espacio agrario (tabla 3).
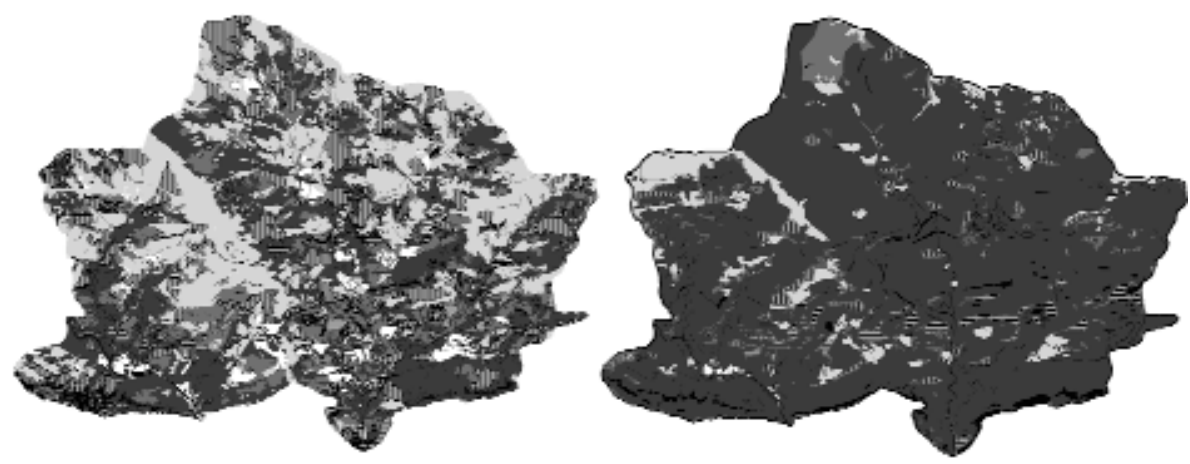

Leyends
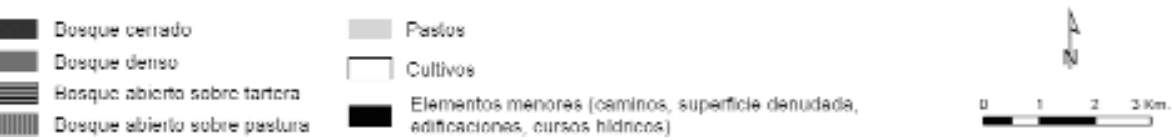

Figura 3. Mapas usos y cubiertas del suelo de 1957 y 2003.

Figure 3. Land cover and land use maps between 1957 and 2003. 
J. VILA SUBIRÓS, A. RIBAS PALOM, D. VARGA LINDE \& A. LLAUSÀS PASCUAL

Tabla 3. Usos y cubiertas del suelo en el año 2003. Table 3. Land use and land cover in 2003.

\begin{tabular}{|lcc|}
\hline Usos y cubiertas del suelo & Hectáreas & $\%$ \\
\hline Bosque cerrado & 4094,02 & 79,65 \\
Bosque denso & 86,68 & 1,69 \\
Bosque abierto sobre pasto & 294 & 5,72 \\
Bosque abierto sobre canchal & 156,34 & 3,04 \\
Cultivos & 5,59 & 0,11 \\
Superficie denudada & 118,80 & 2,31 \\
Edificaciones & 1,08 & 0,02 \\
Pastos & 334,47 & 6,51 \\
Pistas forestales & 21,84 & 0,42 \\
Superficie fluvial & 27,25 & 0,53 \\
\hline TOTAL & $\mathbf{5 1 4 0 , 0 9}$ & $\mathbf{1 0 0 , 0 0}$ \\
\hline
\end{tabular}

Fuente: Elaboración propia a partir de las ortofotografías del ICC, E/ 1:5000 del año 2003.

La comparativa sobre la evolución de los usos y cubiertas del suelo entre los años 1957 y 2003 (figura 3 y tabla 4) pone de relieve de forma aún más clara el proceso de homogeneización del paisaje que ha experimentado este territorio. El dato más relevante es el incremento del 140\% del bosque cerrado, así como la desaparición del $74 \%$ de los pastos y el $96 \%$ de las tierras agrícolas respecto a la superficie ocupada en el año 1957.

Tabla 4. Evolución de usos y cubiertas del suelo normalizados (1957-2003). Table 4. Normalized land use and land cover evolution (1957-2003).

\begin{tabular}{|lcc|}
\hline Usos y cubiertas del suelo & Hectáreas & $\%$ \\
\hline Bosque cerrado & 2393,61 & 140,76 \\
Bosque denso & $-580,30$ & $-87,00$ \\
Bosque abierto sobre pasto & $-493,93$ & $-62,68$ \\
Bosque abierto sobre canchal & 2,45 & 1,59 \\
Cultivos & $-149,05$ & $-96,38$ \\
Superficie denudada & $-0,67$ & $-0,56$ \\
Edificaciones & $-1,37$ & $-55,75$ \\
Pastos & $-1112,04$ & $-76,87$ \\
Pistas forestales & $-63,93$ & $-74,53$ \\
Superficie fluvial & $-0,02$ & $-0,06$ \\
\hline
\end{tabular}

Fuente: Elaboración propia. 
Otro interesante análisis es la determinación de los cambios en los usos y cubiertas del suelo tomando como punto de partida los existentes en el año 1957. La tabla 5 pone de relieve un mantenimiento del bosque cerrado del 1957 en el 2003 (90\% de los casos), así como la evolución del bosque denso y abierto sobre pasto hacia el bosque cerrado $(83 \%$ y $80 \%$ del total, respectivamente). Esta dinámica es parecida también en el caso de los pastos con una clara dinámica de evolución hacia el bosque cerrado $(73 \%)$ y en menor medida en los cultivos (57\%) que también han sido reconvertidos en algunos casos en nuevas zonas de pasto $(23 \%)$ o bosque abierto sobre pasto (16\%).

Tabla 5. Matriz de cambios de los usos y cubiertas del 1957 respecto a los usos y cubiertas del suelo del año 2003.

Table 5. Transition matrix in 1957 regarding land use and land cover in 2003.

\begin{tabular}{|c|c|c|c|c|c|c|c|c|}
\hline Usos 2003 & Bosque & Bosque & Bosque abierto & Bosque abiertc & Cultivos & Superficie & Pastos & Otros \\
\hline Usos 1957 & & & & & & & & \\
\hline Bosque cerrado & 89,6 & 2,21 & 2,92 & 2,32 & 0 & 0 & 2,67 & 0,28 \\
\hline Bosque denso & 82,69 & 3,53 & 4,42 & 6,57 & 0 & 0 & 2,66 & 0,13 \\
\hline $\begin{array}{l}\text { Bosque abierto } \\
\text { sobre pasto }\end{array}$ & 79,42 & 6,81 & 5,64 & 0 & 0 & 0 & 7,82 & 0,31 \\
\hline $\begin{array}{l}\text { Bosque abierto } \\
\text { sobre canchal }\end{array}$ & 68,37 & 0 & 0 & 24,6 & 0 & 7,03 & 0 & 0 \\
\hline Cultivos & 57,05 & 0 & 16,23 & 0 & 3,5 & 0 & 23,22 & 0 \\
\hline Pastos & 73,34 & 1,97 & 11,74 & 0 & 0 & 1,21 & 11,74 & 0 \\
\hline
\end{tabular}

Fuente: Elaboración propia.

Desde una perspectiva de análisis a partir de índices de ecología del paisaje de la evolución de las distintas clases, es decir de cada uno de los tipos de usos y cubiertas del suelo con variaciones más significativas, se puede obtener una interesante información sobre las dinámicas experimentadas por la estructura del paisaje.

Los resultados recopilados en la tabla 6 muestran un importante incremento de la superficie media de las teselas (MPS) de bosque cerrado fruto de su propia expansión hasta llegar a constituir una única tesela de 4084,83 ha. Asimismo se ha producido una reducción del número de teselas en relación a la superficie del área de estudio (PD) y de la densidad de ecotonos (ED). Estos datos ponen claramente de relieve el galopante proceso de homogeneización paisajística de base forestal. Una dinámica que tiene al mismo tiempo como contrapartida la reducción de la superficie media de las teselas (MPS) ocupadas por espacios abiertos (bosque sobre pasto, pastos y zonas agrícolas) 
fruto del proceso de fragmentación al que están sometidos. Esta tendencia supone a la vez un incremento del número de teselas por unidad de superficie (PD) pero que cada vez son de menor tamaño, de la misma manera que implica una reducción de la densidad de ecotonos al ser menor su presencia absoluta en relación a la superficie (ED) (Figura 2).

Tabla 6. Cambios en la estructura de las clases de teselas (1957-2003). Table 6. Changes in the structure of match categories (1957-2003).

\begin{tabular}{|c|c|c|c|}
\hline Clase & $\triangle M P S$ & $\triangle P D$ & $\Delta E D$ \\
\hline Bosque cerrado & 4084,83 & $-3,6$ & $-57,7192$ \\
\hline Bosque denso & 1,8 & $-4,81$ & $-79,0466$ \\
\hline $\begin{array}{l}\text { Bosque abierto sobre } \\
\text { canchal }\end{array}$ & $-1,31$ & 1,08 & $-11,6774$ \\
\hline $\begin{array}{l}\text { Bosque abierto sobre } \\
\text { pasto }\end{array}$ & $-3,61$ & 2,99 & $-59,243$ \\
\hline Cultivos & $-0,26$ & $-2,41$ & $-9,8107$ \\
\hline Pastos & $-4,28$ & 1,1 & $-54,0084$ \\
\hline
\end{tabular}

MPS: Superficie media de las teselas (Mean Patch Size)

PD: Densidad de teselas (Patch Density).

ED: Densidad de ecotonos (Edge Density).

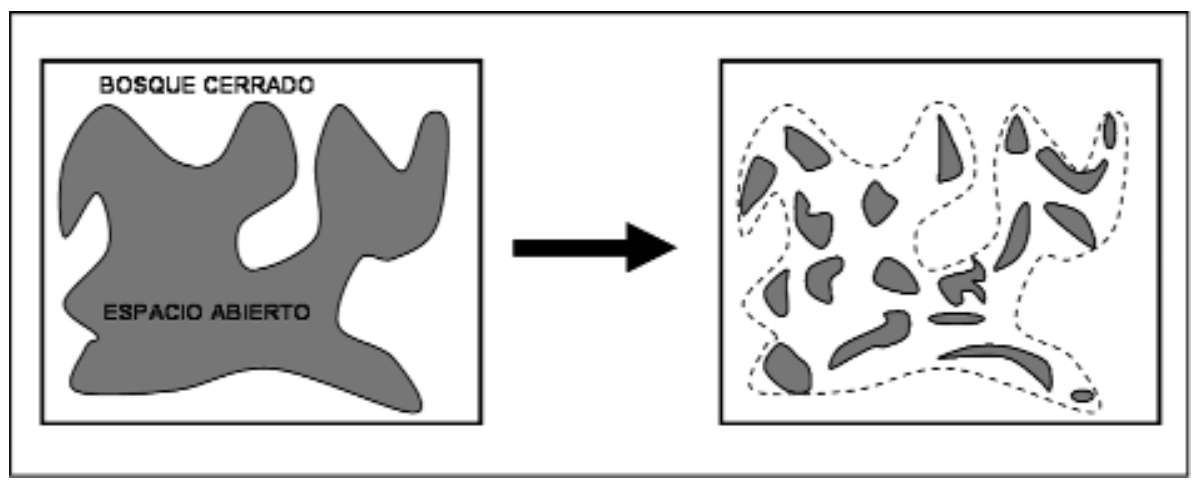

Figura 2. Esquematización del proceso de fragmentación del bosque abierto y los pastos. Figure 2. Diagram of the fragmentation process of open woodlands and pastures. Fuente: Elaboración propia.

En la tabla 7 se pone también de manifiesto el impacto del proceso de homogeneización de base forestal y la fragmentación de los espacios abiertos (bosque sobre pasto, pastos y cultivos). En relación al bosque cerrado la homogeneización implica que las formas evolucionan reduciendo las irregu- 
laridades, tal y como pone de manifiesto la disminución del índice de complejidad de la forma (PAFRAC). A la vez, la presencia de mayor superficie de bosque implica una reducción de la distancia entre las teselas forestales (ENN) y por tanto un incremento de la cohesión (COHESION) debido a que todos los fragmentos son mucho más próximos los unos de los otros. Contrariamente los denominados espacios abiertos responden a la tipología de un proceso de fragmentación con un incremento del índice de complejidad de la forma de las teselas (PAFRAC) que han pervivido y que están bajo la presión del incremento de la superficie forestal generándose formas más irregulares. Al mismo tiempo se detecta una reducción de la cohesión (COHESION) y un incremento de la distancia entre las teselas más próximas (ENN) debido a que la fragmentación supone una mayor separación física entre las teselas presentándose por tanto menos cohesionadas (figura 3).

Tabla 7. Cambios en la forma y conectividad de las clases de teselas 1957-2003. Table 7. Changes in the shape and conectivity of the match classes 1957-2003.

\begin{tabular}{|lccc|}
\hline Clase & $\boldsymbol{\Delta P A F R A C}$ & $\boldsymbol{\Delta}$ ENN & $\boldsymbol{\Delta C O H E S I O N}$ \\
\hline Bosque cerrado & $-0,086$ & $-19,13$ & 0,4068 \\
Bosque denso & 0,0117 & 921,52 & $-0,1226$ \\
Bosque abierto sobre canchal & $-0,0165$ & 58,83 & $-2,2288$ \\
Bosque abierto sobre pasto & 0,0834 & $-19,24$ & $-5,4739$ \\
Cultivos & 0,1405 & 466,84 & $-0,2186$ \\
Pastos & 0,1242 & 80,29 & $-1,582$ \\
\hline
\end{tabular}

PAFRAC: Perímetro-área y dimensión fractal: índice de complejidad de la forma (perimeter-área fractal dimensión).

ENN: $\quad$ Distancia a la tesela más próxima (nearest-neighbor distance)

COHESION: Cohesión entre teselas (patch cohesion index).

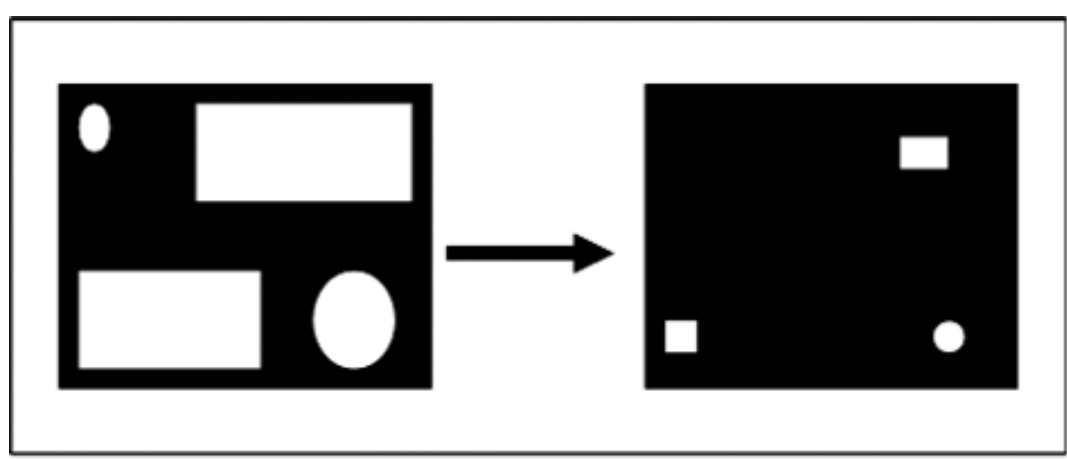

Figura 3. Esquematización del proceso de aislamiento y disgregación de los espacios abiertos. Figure 3. Diagram of isolating process of open areas. 
Finalmente el cálculo del índice de diversidad de Shannon (SHDI) aporta información relevante en relación a la evolución de la heterogeneidad de los paisajes entre los dos momentos temporales objeto de estudio (tabla 8). Los resultados para el Alta Garrotxa ponen de relieve una clara reducción de la diversidad paisajística ya que ésta ha disminuido prácticamente en más de dos tercios a lo largo del periodo estudiado (figura 4).

Tabla 8. Evolución del índice de diversidad de Shannon (SHDI) (1957-2003). Table 8. Evolution of the Shanon Diversiy Index (SHDI) (1957-2003).

\begin{tabular}{|lc|}
\hline & SHDI \\
\hline Paisaje 1957 & 1,66 \\
Paisaje 2003 & 0,52 \\
Paisaje 1957-2003 & $-1,14$ \\
\hline
\end{tabular}

Fuente: Elaboración propia.

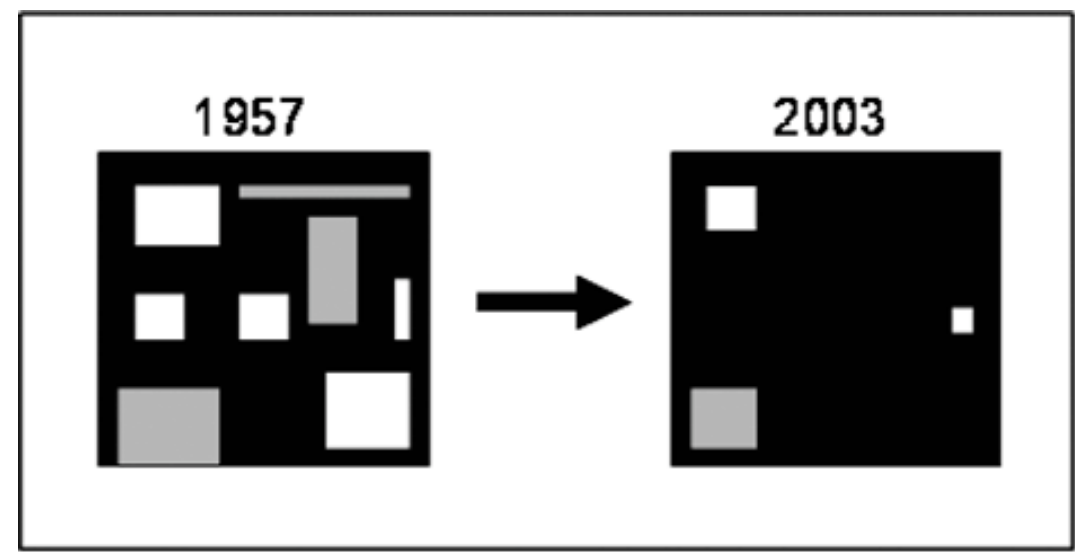

Figura 7. Esquematización del proceso de homogeneización y pérdida de heterogeneidad paisajística. Fuente: elaboración propia.

Figure 7. Diagram of the process of homogenization and loss of landscape diversity.

4.2. Análisis de la percepción y valoración social de los cambios en el paisaje y sus efectos

En este apartado se analiza la relación existente entre la dinámica del paisaje que se ha constatado a lo largo del período 1957 - 2003 (evolución de los 
usos y cubiertas del suelo y aplicación de índices de paisaje) con la percepción y valoración de estas mismas dinámicas por parte de los agentes sociales vinculados al área de estudio. El objetivo es en primer lugar constatar si existe coincidencia entre los cambios paisajísticos estudiados en el apartado anterior y la percepción que de estos tienen los agentes sociales vinculados a la Alta Garrotxa, y en segundo lugar analizar la valoración (positiva, negativa o ambivalente) que de estos cambios realizan estos mismos agentes sociales.

Tabla 9. Dinámicas del paisaje constatadas por los diferentes agentes sociales entrevistados. Table 9. Landscape dynamic indicated by the interviewees.

\begin{tabular}{|c|c|c|c|c|c|c|c|c|c|c|c|c|c|c|c|}
\hline \begin{tabular}{l|} 
Dinámicas \\
del paisaje / \\
Agentes sociales
\end{tabular} & $\Delta / \nabla$ & 1 & 2 & 3 & 4 & 5 & 6 & 7 & 8 & 9 & 10 & 11 & 12 & 13 & 14 \\
\hline \multirow{2}{*}{$\begin{array}{l}\text { Superficie } \\
\text { forestal }\end{array}$} & $\Delta$ & & & & & & & & & & & & & & \\
\hline & $\nabla$ & & & & & & & & & & & & & & \\
\hline \multirow[t]{2}{*}{ Pastos } & $\Delta$ & & & & & & & & & & & & & & \\
\hline & $\nabla$ & & & & & & & & & & & & & & \\
\hline \multirow[t]{2}{*}{ Cultivos } & $\Delta$ & & & & & & & & & & & & & & \\
\hline & $\bar{\nabla}$ & & & & & & & & & & & & & & \\
\hline \multirow{2}{*}{$\begin{array}{l}\text { Patrimonio } \\
\text { cultural }\end{array}$} & $\Delta$ & & & & & & & & & & & & & & \\
\hline & $\nabla$ & & & & & & & & & & & & & & \\
\hline \multirow{2}{*}{$\begin{array}{l}\text { Caminos y } \\
\text { pistas forestales }\end{array}$} & & & & & & & & & & & & & & & \\
\hline & $\bar{\nabla}$ & & & & & & & & & & & & & & \\
\hline \multirow{2}{*}{$\begin{array}{l}\text { Biodiversidad } \\
\text { forestal }\end{array}$} & $\Delta$ & & & & & & & & & & & & & & \\
\hline & $\bar{\nabla}$ & & & & & & & & & & & & & & \\
\hline \multirow{2}{*}{$\begin{array}{l}\text { Biodivesidad } \\
\text { espacios abiertos }\end{array}$} & $\Delta$ & & & & & & & & & & & & & & \\
\hline & $\bar{\nabla}$ & & & & & & & & & & & & & & \\
\hline
\end{tabular}

1: Agricultor y ganadero autóctono; 2: Agricultor y ganadero no autóctono; 3: Agricultor y ganadero no autóctono de origen centroeuropeo; 4: Administración pública- representante político; 5: Administración pública- representante técnico, 6: Propietario forestal; 7: Cazador; 8: Grupo ecologista; 9: Investigador; 10: Excursionista; 11: Asociación cultural; 12 : Artista; 13: Turismo rural y 14: Empresario deportes de aventura.

1: Native farmer; 2: Not native farmer; 3: Not native farmer from Central European origin, 4: Public administration politician; 5: Civil service technician; 6: Forest owner, 7: Hunter, 8: Ecologist association; 9: Researcher; 10: Hiking association; 11: Cultural association; 12: Artist; 13: Touristic farmhouse and 14: Adventure sports businessman.

Las transformaciones paisajísticas más unánimemente constatadas por los entrevistados guardan relación con el aumento de la superficie forestal, la reducción de los pastos y el aumento de pistas forestales (tabla 9). En concreto 13 de los 14 agentes sociales entrevistados han percibido un incremento de la superficie forestal; una dinámica acompañada al mismo tiempo por una 
reducción de los pastos. El agente social que no ha detectado estos cambios en relación al bosque y pastos se dedica a los deportes de aventura, una actividad de reciente implantación en el territorio, lo que seguramente explica su menor apreciación de las transformaciones del paisaje en los últimos cincuenta años. Asimismo 13 de los 14 agentes sociales han destacado el incremento en el número de pistas forestales, y solamente el representante de los cazadores no considera significativo este parámetro. En cambio el número se reduce hasta 11 de los 14 entrevistados cuando se trata de apreciar la reducción experimentada por el patrimonio cultural y la superficie agrícola de la Alta Garrotxa. En el caso de la superficie agrícola esta es una constatación esperable pues siempre ha tenido un papel bastante marginal debido a las propias características geográficas de la Alta Garrotxa. Finalmente sólo 8 de los 13 entrevistados han percibido cambios en relación a la biodiversidad, tanto la forestal como la vinculada a los espacios abiertos.

Por lo que se refiere a la valoración que los entrevistados realizan de estas dinámicas paisajísticas observadas (tabla 10), la mayor parte de ellos valoran como negativo el incremento de la superficie forestal, en concreto 9 de los 14 entrevistados. Pero un número significativo ( 3 de ellos), valoran positivamente esta dinámica. En relación a la disminución de los pastos existe una valoración unánime en sentido negativo entre los 11 agentes que destacan este aspecto y lo mismo ocurre en el caso de la superficie agrícola y el patrimonio cultural. Más reñida es la valoración en relación al incremento de las pistas forestales ya que de las 12 valoraciones realizadas 6 tienen una consideración positiva y 6 una consideración negativa. La evolución de la biodiversidad forestal es valorada positivamente por la mayoría de los agentes que destacan este aspecto, en concreto 6 de 7. En cambio se considera negativa en el caso de los espacios abiertos entre los 6 agentes que han referenciado esta valoración.

Por último, y por lo que se refiere a la valoración de las acciones y políticas implementadas, proyectadas o desarrolladas por distintas administraciones públicas (tabla 11), se pone de relieve una máxima complejidad en la valoración que realizan los distintos agentes sociales entrevistados en relación a estas acciones. En concreto 6 de los 14 agentes consideran positivo el actual nivel de protección de este territorio en el marco del Plan de Espacios de Interés Natural (PEIN), al mismo tiempo que 6 de los 14 tienen una valoración ambivalente, es decir algunos aspectos son considerados positivos y otros negativos, y 3 de los 14 lo valoran negativamente. En relación al actual modelo de gestión estructurado a partir del organismo de gestión propio que es el Consorci de l'Alta Garrotxa, 5 de los 14 entrevistados lo valoran positivamente, 6 lo valoran de forma ambivalente y 3 de manera negativa. El papel de los ayuntamientos es tomando en consideración sólo por 4 de los entre- 
MEDIO SIGLO DE CAMBIOS PAISAJÍSTICOS EN LA MONTAÑA MEDITERRÁNEA. PERCEPCIÓN...

Tabla 10. Valoración de las dinámicas del paisaje constatadas por los diferentes agentes sociales entrevistados (negro: valoración negativa, tramado: valoración positiva y blanco: sin valoración). Table 10. Valuation of the landscape dynamic indicated by the different interviewee (black: negative valuation, gray: positive valuation, white: no valuation).

\begin{tabular}{|l|l|l|l|l|l|l|l|l|l|l|l|l|l|l|}
\hline $\begin{array}{l}\text { Dinámicas } \\
\text { del paisaje } \\
\text { Agentes sociales }\end{array}$ & 1 & 2 & 3 & 4 & 5 & 6 & 7 & 8 & 9 & 10 & 11 & 12 & 13 & 14 \\
\hline $\begin{array}{l}\text { Superficie } \\
\text { forestal }\end{array}$ & & & & & & & & & & \\
\hline Pastos
\end{tabular}

Tabla 11. Valoración por parte de los diferentes agentes sociales de las acciones y políticas realizadas o proyectadas en Alta Garrotxa (negro: valoración negativa; tramado: valoración positiva y gris: valoración ambivalente y blanco: sin valoración).

Table 11. Valuation of the land management or land cover projects in the Alta Garrotxa. (black: negative valuation, gray: positive valuation, white: no valuation).

\begin{tabular}{|l|l|l|l|l|l|l|l|l|l|l|l|l|l|l|}
\hline $\begin{array}{l}\text { Valoración } \\
\text { acciones y } \\
\text { políticas/Agen- } \\
\text { tes sociales }\end{array}$ & 1 & 2 & 3 & 4 & 5 & 6 & 7 & 8 & 9 & 10 & 11 & 12 & 13 & 14 \\
\hline $\begin{array}{l}\text { PEIN (Plan de } \\
\text { Espacios de } \\
\text { Interés Natural) }\end{array}$ & & & & & & & & & & & & & & \\
\hline $\begin{array}{l}\text { Consorci Alta } \\
\text { Garrotxa }\end{array}$
\end{tabular}


vistados, con 3 valoraciones negativas y 1 ambivalente. Esta menor toma en consideración del papel de los ayuntamientos responde en parte a la interpretación de que tienen una capacidad de actuación e intervención muy limitada frente a les muchas problemáticas que convergen en este territorio. Finalmente la posibilidad de convertir el Alta Garrotxa en un Parque Natural, lo que supone la implementación de una figura de protección especial de rango superior, es valorada positivamente por 6 de los agentes, de forma ambivalente por 7 y negativamente por 3 .

\section{Conclusiones}

En primer lugar la evolución de los usos y cubiertas del suelo en el periodo 1957-2003 pone de manifiesto un claro proceso de homogeneización del paisaje caracterizado por un extraordinario incremento de la superficie forestal en detrimento de los espacios abiertos (pastos y campos de cultivo). Una dinámica paisajística con una relación directa con el proceso de despoblamiento rural que ha vivido este territorio en las últimas décadas. El resultado final es la implementación de un patrón paisajístico dominado de forma casi exclusiva por una única cubierta del suelo: el bosque. Esta nueva realidad paisajística tiene consecuencias directas a nivel biológico, cultural y en la incidencia potencial de riesgos naturales como los incendios forestales.

En segundo lugar cabe destacar que la comparativa entre la cuantificación de los cambios en los usos y cubiertas del suelo en el período estudiado coincide en gran medida con la percepción de los agentes sociales implicados en la gestión de este espacio natural protegido. Esta afirmación es especialmente evidente en el caso de aquellas dinámicas que tienen una incidencia morfológica en el paisaje como sería el incremento de la superficie forestal, la reducción de los pastos, la práctica desaparición de los cultivos o la degradación del patrimonio cultural. En cambio las alteraciones de base ecológica, como la dinámica de la biodiversidad forestal y de los espacios abiertos, que necesitan de una mirada más especializada, son percibidas en menor medida por los agentes.

En tercer lugar, por lo que se refiere a la valoración de las dinámicas paisajísticas, los resultados obtenidos en este estudio ponen de manifiesto una doble perspectiva con un alto potencial grado de confrontación ante una eventual toma de decisiones de gestión. Por una parte existe una mayoría de agentes sociales que desde una interpretación de base antropocéntrica valoran negativamente el gran cambio paisajístico que ha supuesto el incremento de la superficie forestal, que vinculan en muchas ocasiones a una naturaleza más salvaje e indómita, fuera del control humano. En cambio, como contrapunto, encontramos una parte significativa de los agentes sociales que desde 
una interpretación de base ecocéntrica valoran positivamente esta evolución, al considerar que responde a los cánones propios de la dinámica y la evolución ecológica natural.

En cuarto lugar cabe remarcar de forma especial las diferencias detectadas en relación a la valoración del incremento de los caminos y pistas forestales por parte de los agentes sociales entrevistados ya que se ponen de relieve visiones contrapuestas en relación a estos cambios. En concreto, la mitad de los entrevistados valoran positivamente este incremento al suponer básicamente una mejora de las comunicaciones viarias ya que el aislamiento geográfico que históricamente ha caracterizado la Alta Garrotxa (debido, entre otras causas, a la deficiente red de comunicaciones viarias existente), ha sido considerada como uno de los elementos que ha favorecido el abandono rural y ha impedido el desarrollo económico y social de este territorio. En contraposición, la otra mitad de los entrevistados hace una valoración negativa de este aspecto por su impacto paisajístico y al considerar que favorece una frecuentación motorizada no deseable para la conservación del patrimonio natural y cultural de este espacio protegido. Así pues, se pone de manifiesto la valoración dual de otro elemento que ha marcado los cambios en el paisaje de la Alta Garrotxa y que, a la vez, se ha convertido en un punto central de conflictividad en relación a su gestión pasada, presente y futura.

Finalmente, cabe destacar que la compleja y diversa valoración que los agentes sociales entrevistados realizan del actual modelo de gestión de este espacio natural protegido, así como las incertidumbres que despiertan los potenciales escenarios de futuro, ponen de relieve que no existe en este momento el mínimo consenso social necesario en relación a las prioridades de gestión territorial a desarrollar. Sin duda alguna se hace necesario fomentar la participación pública y el debate entre todos los agentes sociales involucrados, tanto desde el sector público como del privado, con el objetivo final de favorecer la definición de un modelo de gestión que integre, en la medida de lo posible, las distintas percepciones, valoraciones y sensibilidades sociales en torno al futuro de la Alta Garrotxa.

\section{Agradecimientos}

Este artículo se enmarca en el proyecto Dynamique des paysages, erósion et développement durable dans les montagnes méditerranéennes financiado por el Ministère de l'Écologie et du Développement Durable (Francia) (2005-2008). Asimismo agradecemos sinceramente a Josep Masdevall su excelente trabajo de transcripción de las entrevistas que los autores realizamos a lo largo del verano y el otoño del año 2006. 
J. VILA SUBIRÓS, A. RIBAS PALOM, D. VARGA LINDE \& A. LLAUSÀS PASCUAL

\section{Referencias}

BEGUERÍA, S.; LÓPEZ-MORENO, J. I.; LORENTE, A.; SEEGER, M. \& GARCÍA-RUIZ J. M. (2003) Assessing the effect of climate oscillations and land-use on streamflow in the central Spanish Pyrenees. Ambio, 32(4): 283286.

BLONDEL, J. \& ARONSON, J. (1999). Biology and wildlife of the Mediterranean region. Oxford University Press, 328 pp., Oxford.

BOADA, M. (2003). Boscos de Catalunya. Història y actualitat del món forestal. Brau Edicions, 188 pp., Figueres.

BOTEQUILHA, A.; MILLER, J.; AHERN, J. \& MCGARIGAL, K. (2006). Measuring Landscapes. A Planner's Handbook. Island Press, 245 pp., Washington.

CONSANDEY, C.; ANDRÉASSIN, V.; MARTIN, C.; DIDON-LESCOT, J.F.; LAVABRE, J.; FOLTON, N.; MATHYS, N. \& RICHARD, D. (2005). The hydrological impact of the mediterranean forest: a review of the French research, Journal of Hydrology, 301: 235-249.

DEFRIES, R. \& ESHLEMAN, K.N. (2004). Land-use change and hydrologic processes: a major focus for the future, Hydrological Processes, 18: 21832186.

FABREGÓ, J., MONTSERRAT, J. \& VILA, J. (2008). Seguiment a l'Alta Garrotxa de les poblacions d'ocells incloses a l'annex y de la Directiva Aus: El cas de l'àguila daurada (Aquila chrysaetos). Vila, J. (ed). La conservació del patrimoni natural y la biodiversitat. Càtedra de Geografia y Pensament Territorial y Consorci de 1'Alta Garrotxa. Girona, 101-106.

FARINA, A. (1997). Landscape structure and breeding bird distribution in a sub-Mediterranean agro-ecosystem. Landscape Ecology, 12: 365-378.

FARINA, A.; SANTALONI, R.; PABLIARO, G.; SCOZZAFAVA, S. \& SCHIPANI, I., (2005). Eco-semiotics: A new field of competence for ecology to overcome the frontier between environmental complexity and human culture in the Mediterranean. Israel Journal of Plant Sciences, 53: 167175.

FARINA, A. (2006). Principles and Methods in Landscape Ecology. Towards a Science of the Landscape. Springer, 412 pp., London.

FORMAN, R.T.T. \& GODRON, M., (1986). Landscape Ecology. Wiley \& Sons, 619 pp., New York.

FORMAN, R.T.T. (1995). Land Mosaics: the Ecology of Landscapes and Regions. Cambridge University Press, 632 pp., Cambridge.

GROVE, A. T. \& RACKHAM, O. (2000). The nature of Mediterranean Europe: An ecological history. Yale University Press, 384 pp., New Haven.

HUNZIKER, M.; FELBER, P.; GEHRING, K.; BUCHECKER, M.; BAUER, N.; 
\& KLENAST, F. (2008). Evaluation of landscape change by different social groups. Mountain Research and Development, 28 (2): 140-147.

LASANTA, T. (1988). The process of desertion of cultivated areas in the Centrals Spanish Pyrenees. Pirineos, 132: 15-36.

LASANTA, T.; VICENTE-SERRANO, S. M. \& CUADRAT, J. M. (2005). Mountain mediterranean landscape evolution caused by the abandonment of traditional primary activities: a study of the Spain Central Pyrenees. Applied Geography, 25: 47-65.

LASANTA, T.; GONZÁLEZ-HIDALGO, J. C.; VICENTE-SERRANO, S. M. \& SFERI, E. (2006). Using landscape ecology to evaluate an alternative management scenario in abandoned Mediterranean mountain areas. Landscape and Urban Planning, 78: 101-114.

LEWIS, J. L. (2007). Perceptions of landscape change in a rural Bristish Columbia community. Landscape and Urban Planning, 85: 49-59.

LI, H. Y WU, J. (2004). Use and misuse of landscape metrics. Landscape Ecology, 19: 389-399.

MCGARIGAL, K. \& MARKS, B. (1995). FRAGSTATS: a spatial pattern analysis program for quantifying landscape structure $v 2.0$ (unpublished computer program user manual and guide), Oregon State University,141 pp., Cornallis.

MCGARIGAL, K. \& CUSHMAN, S. A. (2005). The gradient concept of landscape structure. Wiens, J y Moss, M. (eds.) Issues and Perspectives in Landscape Ecology. Cambridge, Cambridge University Press, 112-119.

OTERO, I.; BOADA, M.; BADIA, A. \& PIQUERAS, S. (2008). El canvi global a la conca de la Tordera. Institució Catalana d'Història Natural (ICHN). Els sistemes socioecològics de la conca de la Tordera. ICHN, Barcelona, 95-129.

PAGĖS, M. \& ANGLADA, M. (2008). Inventari de la comunitat d'ungulats salvatges de l'Alta Garrotxa". Vila, J. (ed). La conservació del patrimoni natural y la biodiversitat. Càtedra de Geografia y Pensament Territorial y Consorci de l'Alta Garrotxa. Girona, 115-128.

SWIHART, R. \& MOURE, J. (2004). Conserving Biodiversity in Agricultural Landscapes (Model Based in Planning Tools). Purdue University Press,336 pp., Indiana.

TRABALÓN, F. (2008). Seguiment a l'Alta Garrotxa de les poblacions d'ocells incloses en l'annex y de la Directiva Aus. Vila, J. (ed). La conservació del patrimoni natural y la biodiversitat. Càtedra de Geografia y Pensament Territorial y Consorci de l'Alta Garrotxa. Girona, 89-100.

VARGA, D. \& VILA, J. (2006). Ecología del paisaje y sistemas de información geográfica ante el cambio socioambiental en las áreas de montaña mediterránea. Una aproximación metodológica al caso de los valles de Hortmoier y Sant Aniol (Alta Garrotxa, Girona). AREAS. Revista Internacional de Ciencias Sociales, 25: 59-72. 
J. VILA SUBIRÓS, A. RIBAS PALOM, D. VARGA LINDE \& A. LLAUSÀS PASCUAL

VARGA, D. (2007). Paisatge y abandonament agrari a la muntanya mediterrània. Una aproximació al cas de les valls d'Hortmoier y Sant Aniol (Alta Garrotxa) des de l'ecologia del paisatge, Girona, Tesi doctoral del Programa de Doctorat de Medi Ambient de la Universitat de Girona, 371 pp.

VILA, J. (2000). Anàlisi y valoració dels boscos de les Valls d'Hortmoier y Sant Aniol (Alta Garrotxa), Tesi doctoral, Departament de Geografia Física y Anàlisi Geogràfica Regional, Universitat de Barcelona, $510 \mathrm{pp}$.

VILA, J.; VARGA, D.; LLAUSÀS, A. \& RIBAS, A., (2006). Conceptos y métodos fundamentales en ecología del paisaje (landscape ecology). Una interpretación desde la geografía, Documents d'Anàlisi Geogràfica, 48: 151-166. 